\title{
Conducting Online Focus Groups - Practical Advice for Information Systems Researchers
}

\author{
Laura Schulze \\ University of Goettingen \\ Laura.schulze@uni- \\ goettingen.de
}

\author{
Manuel Trenz \\ University of Goettingen \\ trenz@uni-goettingen.de
}

\begin{abstract}
Video-based online focus groups present an emerging opportunity for IS researchers to collect rich data. They allow researchers to assemble participants from all over the world who collectively discuss contemporary IS phenomena. In order to realize the full potential of online focus groups for IS research, we need to understand the challenges and uncover possible solutions for designing and conducting online focus groups. We review prior (online) focus group literature in and beyond the IS discipline. Additionally, we provide a detailed account of our own experiences with seven online focus groups in the context of digital platforms. In supplementing our own experiences with those of others in prior literature, we present the conditions under which online focus groups are especially appropriate, summarize the challenges inherent in the online focus group method and provide practical advice on its application.
\end{abstract}

\section{Introduction}

"The scientific basis of knowledge does not necessarily lie in the research method; rather, it lies in how we design the research and implement it." [1, p. 147]

Focus groups are employed as a means of qualitative data collection across scientific disciplines [2]. Particularly, in the marketing and health disciplines, focus groups are a common method for eliciting research insights. To a growing extent, focus groups are becoming an indispensable component of the methodological toolkit for information systems (IS) researchers as well [3]. They involve a focused discussion among people who possess knowledge on the topic of interest [4].

Focus groups are invaluable for eliciting people's understandings, opinions and views [2]. Participants express concepts and concerns in their own language [2]. By means of the group discussion, they add more

\author{
Zhao Cai \\ University of Nottingham \\ Ningbo China \\ Zhao.Cai@nottingham.ed \\ u.cn
}

\author{
Chee-Wee Tan \\ Copenhagen Business \\ School \\ ct.digi@cbs.dk
}

depth to the data than individual interviews [5]. Unknown information is uncovered and surfaced [1], and focus groups allow for the production of more fully articulated accounts [2], collective sensemaking [2], and constructing collective views [6].

In research in general, focus groups are conducted for theory development, confirmation, triangulation and evaluation [1]. In IS research specifically, they are employed in isolation, or, in combination with other methods [3], such as case studies, design science research, and scale development. Their purpose is either exploratory, or explanatory [3]. Although focus groups are useful for a broad range of purposes [3], practical restrictions of the face-to-face setting limit their applicability. The need for collocation excludes groups of desired participants, makes focus groups expensive and time consuming [7], [8], and introduces undesired group dynamics [1].

To overcome these limitations, researchers have begun using technology to conduct focus groups online. They can be text-based (e.g., social media, forums or email), audio-based (e.g., telephone, voice messages) or video-based (e.g., videoconferencing) [5]. Prior research found that video-based online focus groups provide " $[\ldots]$ the opportunity for a natural flow of ideas, similar to that achieved in a traditional focus group" [7, p. 1626]. While text-based online focus groups have been subjected to extensive methodological investigations and applications, there is a lack of knowledge within extant literature on video-based online focus groups [9]. In this paper, we refer to online focus groups (OFGs) when they are video-based (unless specified otherwise).

The use of the focus group method is based on the purpose of the study and the nature of the phenomenon of interest [4]. Despite the fit of the focus group method with a particular IS phenomenon under investigation, physical distances and resource limitations might have prevented researchers from applying them. Contrarily, online focus groups eliminate the spatial and temporal boundaries and enable IS researchers to collect rich data 
from distributed participants on any given IS phenomenon. They are especially applicable to contexts in which the phenomenon of interest is global and the population of interest is geographically distributed. There are plenty of examples for such phenomena in the IS field, including digital platforms, social media, global outsourcing, and cross-border project management.

Despite the promise of online focus groups for IS research, practical guidelines on how to conduct them are scarce, but necessary in order to successfully exploit their capabilities to create knowledge (see introductory quote). There are many challenges associated with online focus groups, including for example scheduling.

The purpose of this paper is to elucidate the challenges confronting researchers in designing and conducting online focus groups and put forth advice on how these challenges can be overcome. We draw on our own experiences with online focus groups and present the results in the form of a confessional tale, which "lift[s] the veil of public secrecy surrounding fieldwork" [10, p. 91]. It is a form of fieldwork writing which admits flaws in the account [10]. We believe that this provides other researchers with invaluable learning opportunities and allows them to collectively advance the online focus group method in the future.

\section{Focus groups in prior IS literature}

To investigate how focus groups have been employed in IS research, we conducted a review of extant literature. In line with Wiesche et al. [10], we searched the full texts of 13 leading IS and management journals for 'focus group'.

After skimming through the 571 retrieved articles, we discovered that $305(53 \%)$ of them conducted focus groups. Information \& Management (49), the International Journal of Information Management (47), Management Information Systems Quarterly (38), the European Journal of Information Systems (36) and Decision Support Systems (34) are the journals which published the most studies including focus groups. Between 2011 and 2020, an average of 17 papers per year was published. We found that only 61 (20\%) of them actually reported on the focus group method in detail. This finding resonates with previous literature [3]. The main reason appears to be that focus groups are often conducted in addition to, or as a part of another methodology, such as scale development studies, case studies or design science research projects.

In a review of extant literature, Bélanger [3] found that focus groups, in isolation, are employed for investigating phenomena such as usage and managerial issues of technology and systems [3]. Apart from the investigation of group-level, or relational phenomena, they are also useful for theory building when concepts of interest emerge from the group discussion and when the subjects are embedded in a collective [3].

In isolation, and in conjunction with other methods, focus groups are employed in IS research to generate all five types of theories that Gregor defines [11]: analyzing, explaining, predicting, explaining and predicting, and design and action [3]. In other words, focus groups can be employed for both, exploratory or explanatory purposes [3]. With their broad area of application, focus groups can contribute to theorization in multiple ways.

However, in the past, practical restrictions inherent to the face-to-face setting have rendered focus groups infeasible or unattractive for researchers. Focus group participants and moderators, usually the researchers, have to be collocated at the same place. This not only translates into challenges in terms of sampling, time and costs, [7], [8], but it also introduces bias due to unpredictable group dynamics [1]. It is very expensive and time consuming to get all focus participants to one place, and in some cases, it makes it impossible to conduct focus groups with certain groups of people. For instance, busy professionals and experts, rural communities and people unable to travel due to physical conditions are excluded from participation in focus groups [7], [12]. Their unavailability reduces and biases samples. Additionally, the physical research setting itself can introduce biases in face-to-face focus groups, for instance because of the seating order, personal space and privacy issues [1]. Online focus groups, when done rigorously, may be able to overcome these restrictions.

\section{Online focus groups for investigating IS phenomena}

Beyond their ability to facilitate discussions, online focus groups possess multiple advantages that are not exclusive to any discipline. Thus, we conducted an interdisciplinary literature review and searched ScienceDirect and WebofScience for '(online OR virtual OR video) focus group' in the title/abstract/keywords (ScienceDirect) or topic (WebofScience) sections. Of the 160 retrieved articles, we eliminated false hits, such as duplicates and papers which did not conduct online focus groups, such that we retained 135 papers. We also conducted a backward search. $27 \%$ of papers concerned video-based online focus groups, and $12 \%$ were method papers. The intersection (video-based AND method) was the topic of the five papers we discuss below (i.e. [7]-[9], [12], [13]). We briefly synthesize the general advantages of online focus groups over face-to-face focus groups in the following before turning towards the idiosyncrasies of IS phenomena that advocate the use of this method. 
Online focus groups are cheaper and can be conducted faster than face-to-face focus groups [7], [12], thereby allowing researchers to generate rich insights conveniently and quickly. The applicability of focus groups increases through videoconferencing, because the range of potential participants is global and includes previously hard to reach groups [7], [12]. Additionally, online focus groups are easier to integrate in the usual (work) life [7], which increases peoples' likelihood of participating in an online focus group.

Apart from the broader applicability, online focus group discussions also bear potential for richer insights. Participants are based in their homes or other familiar places where they feel comfortable, anonymous and safe [8], [12]. These feelings contribute to the intended outcomes of focus groups [4], such as an engaged discussion [7], [12]. While the technology mediation reduces some cues, this does not have to be a disadvantage. First, only one participant can speak at a time. This focus group ground rule is easier to enforce through technology and reduces the risk of dominant participants [1]. It allows for more friendly interactions [9] where participants feel respected. Second, unwanted and confounding cues, such as social status [1], are reduced by the online setting and enable more heterogeneity in the composition of focus groups [8].

In our review of the literature, we identified only one paper in the field of IS that employed online focus groups. Bergvall-Kåreborn and Howcroft [14] investigated mobile applications development and distribution. The researchers employed Skype to discuss with two Android developers from Sweden and two Android developers from the UK, respectively. The online focus groups served as additions to face-to-face focus groups and individual interviews. No details are reported about the particularities of online focus groups.

Despite the lack of publications of online focus groups in IS research, we propose that the method will yield valuable insights for the field in the future. The topic of the study and the participant population have to lend themselves to the online focus group method [9].

Topic-wise, the development of information systems, as well as the analysis of interactions of individuals, groups, organizations and markets with technology is at the core of the IS discipline [15]. Within the discipline, there seems to be no topic that would not benefit from the rich data online focus groups can generate, whether it is to generate, or to test theory (see section 2). Especially IS topics in the area of interactions of individuals or groups with technology, such as social analytics, big data, machine learning, augmented reality [16], and personalized technology [17] would benefit from the method.

Participant-wise, geographically dispersed individuals and communities are of interest to IS researchers. For example, chief information officers or project managers in multi-national companies, users of social media, and software developers for mobile applications are typically not collocated in one place and difficult to assemble in one place. Therefore, online focus groups allow studying a broader population of interest.

We summarize the characteristics of the phenomena, as well as those of the participants that make online focus groups especially useful for IS researchers. The more of these characteristics are present, the more appropriate online focus groups are.

(1) Phenomenon is a group interaction, emerges from group interaction, or is surfaced by group interaction, and/ or (a) occurs globally, and/ or (b) is the use of digital technology, and/ or

(2) Participants (a) are geographically dispersed, and/ or (b) restricted in time, and/ or (c) use technology.

We exemplify the application of these characteristics when introducing our online focus group study below (section 4.1). The characteristics apply in cases where online focus groups represent the sole method of data collection, and cases in which they are part of a multi-method study. Apart from situational considerations which might render face-to-face focus groups infeasible, we do not advocate for a replacement of face-to-face focus groups by online focus groups. Rather, we argue for an addition of online focus groups to the researcher's toolkit [8], [12], especially in the cases outlined above.

\section{Conducting online focus groups}

Conducting online focus groups has its own challenges, which we discuss below. In order to realize the full potential of online focus groups for IS research, we provide researchers with advice on how to overcome these challenges. To do so, we draw on our own experiences with online focus groups. To this end, we reference an online focus group study that we recently conducted as an example. A summary of our key challenges and lessons learned is presented in Table 1. We reflect upon our own experiences in light of other researchers who conducted and reported on their experiences with online focus groups in other disciplines. Thereby, we provide practical advice for researchers who plan to design and conduct online focus groups.

\subsection{Exemplary online focus group study}

Study context, research question and purpose: Our study is situated in the context of digital labor platforms, which enable transactions between workers, 
who offer their services, and clients, who desire those services [18]. A typical example of a digital labor platform is Uber - a multinational ridesharing platform connecting local drivers and riders. In order to coordinate the large number of users, digital labor platforms "exercise control through their design features and algorithms, which are a set of rules and routines that are coded and programmed with a set of instructions on how to perform the tasks" [19, p. 5]. The prevalence and impacts of these algorithmic management practices for different types of work and platforms are in the center of our investigation.

Due to the individualization of algorithmic management practices, knowledge about them is scarce. Through collective sensemaking in the group discussion, a deeper understanding of the phenomenon was gained. Based on the characteristics outlined above (see section 3, characteristics 1 a)-c) and 2 a)-c)), we chose online focus groups as the most appropriate and sole method of data collection. The phenomenon is global because most digital labor platforms pander to a worldwide audience (1a). The participants' use of, and experiences with digital labor platforms were investigated (1b). Workers on digital labor platforms are distributed throughout the globe (2a). Oftentimes, they work on these platforms for supplemental income in addition to other responsibilities and can be considered busy (2b). They use the technological interfaces of digital labor platforms (2c), which is also their key common characteristic of interest.

Recruitment and sampling of participants: The population of interest for the online focus groups consists of workers who have substantial work experience on digital labor platforms. Based on our preliminary knowledge from literature we looked for these workers in online forums and groups, where they get in contact with other workers, exchange news, and consolidate. Among others, we used major social media platforms (Reddit, Facebook, LinkedIn, and Baidu Tieba) for online recruitment.

We created a profile for the purpose of the study and requested to join pertinent groups. This included more general groups, e.g., groups for freelancers, and more specific groups, e.g., groups for Clickworker, which is a digital labor platform. Our requests to join were not always accepted by the group administrators.

Between December 2020 and February 2021, we sequentially posted at least once on 52 different forums/ groups. Some forum/ group administrators declined our post, such that it was only published in 32 forums/ groups. In our post, we highlighted non-monetary (e.g., opportunity for exchange with other workers) and monetary benefits for participants and redirected them to a screening survey.
Prior to starting the screening survey, interested workers had to review the privacy statement and give their consent. Afterwards, they were asked about their work experience with different digital labor platforms, demographics, and their availability for the online focus group sessions. Potential participants who were interested in participating in the online focus groups provided their email address, or instant messenger contact details (i.e., WeChat and QQ). After completing the screening survey, they received information on the further process. In sum, we were able to spark the interest of 174 workers worldwide.

After conducting a pilot online focus group with seven participants who were highly diverse in terms of the nature of work they completed on different digital labor platforms, we decided to sample the subsequent groups as follows. Foremost, we grouped them based on the nature of work they performed on the several platforms they have worked with. For this single characteristic, we strived for homogeneity, because we expected from the pilot online focus group that uncovering algorithmic management practices across multiple types of work was more feasible from the cross-group comparison. Then, we screened out all participants without substantial work experience on the platforms, those who indicated a low willingness to contribute to the discussion, and those who spoke none of the languages fluently that we were able to facilitate. Lastly, we compared the availability options they indicated in the screening survey and chose a time that fitted most participants.

Then, we informed selected participants of their admission to the online focus group. When participants were spread throughout different time zones, this email/ message also included the date and time in all relevant time zones and an attachment with a calendar entry. They were asked to save two hours for the meeting. The email/ message also summarized the topic and introduced the moderator. We introduced the videoconferencing tool we used (Zoom/Tencent Meeting) and attached further instructions on the videoconferencing tool at the end of the email/message. We gave participants the meeting link to the videoconference and asked them to be in an environment in which they could use their video and audio without disruptions at the time of the online focus group. We also included two calls for actions. First, we asked them to respond to our email/ message within the next few days. Second, we asked them to access the videoconference via the link at the time of the online focus group. We briefly outlined organizational issues as well. Participants were informed that they will receive further details on their compensation, and technical assistance, including how to change settings ensuring privacy. 
All participants who confirmed their participation received a reminder a few hours before the online focus group started which, again, included the time and the link to the videoconference.

In sum, we invited 68 participants of whom 32 confirmed their participation. Finally, 23 actually joined one of the seven online focus groups (two to seven participants per group). They were between 19 and 44 years old and from nine different countries on four different continents. One online focus group did not take place at all, as none of the participants confirmed their participation. We tried to reschedule the group and asked participants to renew their availability indication based on a time scheduling survey. However, none of these invited participants responded.

Online focus group procedure: The online focus groups were conducted between January 4 and April 17, 2021. One researcher in our author team, who was located in Germany, moderated four online focus groups with international participants. Another researcher in our author team, who was located in China, moderated three online focus groups with participants from China.

At the beginning of the international online focus groups, the moderator was in the main videoconferencing room and welcomed the participants. Right after a participant joined, the moderator redirected her/him to an individual breakout room (they allow moderators to split the videoconference meeting in separate sessions with selected participants) in which another researcher or research assistant was waiting. All research assistants were briefed beforehand to guide the participants through the individual welcoming process.

This process included checking whether sound and audio were working fine, familiarizing participants with the videoconferencing tool, the option to change privacy settings, such as setting an alias or a background filter, giving more details on the payment process and asking for participants' consent to the recording of the session.

Meanwhile the moderator stayed in the main videoconference room, welcomed, and distributed new participants to breakout rooms, and casually talked with participants who were already finished with the individual session. In the Chinese online focus groups, individual sessions took place before the actual online focus groups.

As soon as every participant was welcomed, and it was unlikely that more participants would show up, the participants were asked again to consent to the recording. After obtaining agreement from all participants, the moderator started the built-in recording feature of the videoconferencing tool. Then, the moderator shortly introduced the research team, the research project and set the ground rules for the discussion via videoconference. The introductory questions asked the participants for a short self- introduction along three simple questions. Afterwards, the moderator went through the moderator's guide which contained questions along the work process on the digital labor platforms. The sessions closed with closing questions and opportunities for the participants to raise any additional points or questions.

Due to requirements by one of our universities, it was necessary to follow up with the participants in the four international groups, because they needed to fill out a form in order to process payment. The follow-up email included the form, as well as a suggestion for an individual follow-up meeting a few days after the online focus group. All invited participants arranged for and took part in the individual debriefing meeting. The online focus group videos were transcribed verbatim. The few days in between the online focus group and the individual debriefing allowed us to watch the video and/ or read the transcript and prepare specific follow-up questions for each participant individually.

During the follow-up interview, we also asked for the participants' feedback on the online focus group. Most participants expressed that they enjoyed the interactive group discussion. One participant claimed:

"It was fun. I was glad I was able to contribute as much as I was. I thought I was just going to be sitting there not saying anything."

Participants also found it valuable to exchange their views with other workers and learn from their experiences. One participant claimed:

"Depending on a specific person, the experience is very different if you can share information like that and talk about it, I think it's a benefit for us."

These exemplary quotes reinforce the appropriateness of the online focus groups method in our study. Participants' recommendations for improvement are included in our discussion below. The analysis of the online focus group transcripts is in progress and the results of our study will be presented elsewhere.

\subsection{Practical recommendations for conducting online focus groups}

We structure our discussion of the challenges and possible solutions throughout the steps of a focus group. They include the objectives of the study, the identification and recruitment of participants, the research setting, preparation of the focus group, facilitation of the focus group, data analysis and reporting [6]. We only discuss the steps in which we identify differences for online focus groups, as opposed to face-to-face focus groups. In addition to recruitment and sampling, research setting, preparation, and facilitation, we introduce the follow-up step which takes place after the online focus group. 
Table 1 presents an overview of the key challenges and lessons learned that we derive directly from our own experiences. In the discussion below, we supplement the discussion with accounts and advice that we identified in interdisciplinary online focus group literature.

Table 1. Key challenges and lessons learned

\begin{tabular}{|c|c|c|}
\hline Step & Key challenges & Lessons learned \\
\hline 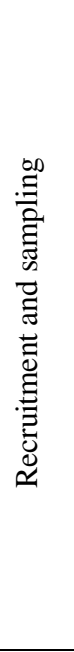 & $\begin{array}{l}\text { - } \begin{array}{l}\text { Recruitment of } \\
\text { anonymous } \\
\text { participants in } \\
\text { online groups/ } \\
\text { forums }\end{array} \\
\text { - Scheduling } \\
\text { issues } \\
\text { - High attrition } \\
\text { - Group } \\
\text { composition }\end{array}$ & $\begin{array}{l}\text { - Adhere to group guidelines; } \\
\text { ask administrators when in } \\
\text { doubt } \\
\text { - Include participants' benefits, } \\
\text { link to screening survey and } \\
\text { university affiliation in post } \\
\text { - Moderator: approximate } \\
\text { participants' time zones } \\
\text { - Coordinate participants' time } \\
\text { preferences in a spreadsheet } \\
\text { - Fast recruitment process; } \\
\text { multiple points of contact } \\
\text { - Substantial over-recruitment } \\
\text { - Single-category design; } \\
\text { determined via screening } \\
\text { survey } \\
\text { - Aim for 3-4 participants per } \\
\text { group }\end{array}$ \\
\hline 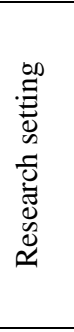 & $\begin{array}{l}\text { - Location } \\
\text { inappropriatene } \\
\text { ss }\end{array}$ & 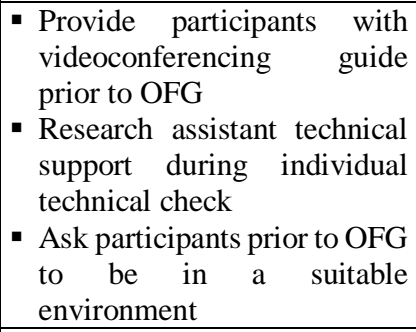 \\
\hline 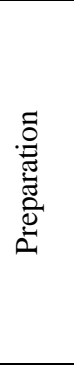 & $\begin{array}{l}\text { - Limited } \\
\text { individual } \\
\text { exchange }\end{array}$ & $\begin{array}{l}\text { - Maintain contact through the } \\
\text { same address throughout the } \\
\text { study } \\
\text { - Verify contact information in } \\
\text { individual welcoming session } \\
\text { - Steady, but unobtrusive flow } \\
\text { of communication } \\
\text { - Individual welcoming } \\
\text { session; conducted by } \\
\text { research assistants } \\
\end{array}$ \\
\hline 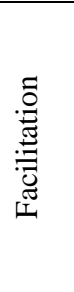 & $\begin{array}{l}\text { - Only one } \\
\text { participant can } \\
\text { speak at a time } \\
\text { - Risk of group } \\
\text { interview }\end{array}$ & $\begin{array}{l}\text { - More active role of the } \\
\text { moderator in turn-taking, } \\
\text { e.g., calling upon specific } \\
\text { participants } \\
\text { - More active role of moderator } \\
\text { in facilitating, e.g., } \\
\text { establishing connection } \\
\text { between comments }\end{array}$ \\
\hline 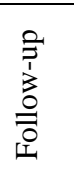 & $\begin{array}{l}\text { - Limited } \\
\text { individual } \\
\text { exchange }\end{array}$ & $\begin{array}{l}\text { - Conduct individual follow-up } \\
\text { interviews; opportunity to ask } \\
\text { clarification questions }\end{array}$ \\
\hline
\end{tabular}

Recruitment and sampling of participants: The goal of the recruitment process is to assemble focus groups with knowledgeable participants on the research topic [4]. Conducting more than one focus group is necessary in order to reach theoretical saturation [20]. When the geographically dispersed participants are unknown to the researchers, online recruitment is likely. However, it is accompanied by some challenges.

Reaching participants online can be difficult and is, as always, subject to self-selection. Our posts in forums/ groups were sometimes regarded as spam by administrators and members. However, the recruitment overall was quite successful as the forums/ groups we chose were heavily populated by our population of interest and members responded positively to our posts.

We advise researchers to pay attention to the community guidelines of the online forums/groups in order to reach participants and avoid upsetting group members. Otherwise, researchers might be banned from the forums/ groups, thus eliminating chances of reaching participants. When in doubt, we found it to be very helpful to contact the group administrators and ask for their permission to post. Care should be taken with regards to specific keywords in the post that could result in automatic declines of posts. In the post, the benefits for the participants should be outlined clearly and a link to the screening survey should be included. To satisfy research ethics standards and to increase credibility among forum/ group members, the researchers' identities and the university affiliation should be clearly stated.

Differences in time zones create obstacles in scheduling the online focus group in the first place [9]. Care should be taken, such that the risk of losing valuable potential participants is minimized. On the researcher side, all efforts should be taken in order to arrange for the most convenient time for participants. In this sense, it is desirable that researchers who moderate the online focus groups match the participants' time zones. In our online focus groups, we were able to approximate this match by dividing up the moderator's role in a strategic way, i.e., a researcher, who was located in China, conducted the all-Chinese online focus groups. If inapplicable, researcher moderators might need to accommodate online focus groups outside usual working hours.

There are different options to overcome scheduling challenges on the participant side. When participants are less committed, it makes sense for the researcher to coordinate scheduling among them. From the online focus group which we planned, but failed to execute due to non-responsive participants, we observed this lack of engagement. We gave the nine selected participants the opportunity to reschedule and provided them with a link to a scheduling software page. However, none of the 
potential participants indicated their availability on the page. Therefore, maintaining a spreadsheet that includes individual time preferences, along with the time zones of the participants [7], [9] seems to be preferable when participants are still less engaged. While our experience was that this was a time-consuming task, it did ease organizational issues for participants and made it more likely for them to actually participate. When all desired participants already consented to participating and are highly engaged, all group members can be asked for their time preferences, using scheduling software [9]. When there are many alternative potential participants readily available, a self-selection of participants to a predefined time might also make sense [12].

Even when an online focus group was successfully scheduled, high attrition rates show that participants frequently drop out throughout the recruitment process. While group composition is a central aspect to focus groups [1], designing them in a pre-defined manner becomes increasingly difficult in online focus groups. Participants show lower commitment to online focus groups [7], [12]. Just like in other online focus group studies, our attrition rate was quite high $(15 \%-67 \%$, without the failed group). The range indicates that, although the process was the same for all groups, there were variations in attendance rates. While sampling challenges exist, countermeasures can be taken.

From the online focus group that failed to take place, we learned that it is crucial to have a short time span between recruitment and execution of the online focus group. Most of the selected participants filled in the screening survey mid December 2020. We only notified them of their acceptance to the online focus group by mid-January. A four weeks' time delay seemed to put participants off. Therefore, the faster lead time of online focus groups [12] has to be taken into account. Participants need multiple points of contact prior to the online focus group in order to actually show up [12].

Regarding group composition, Krueger and Casey [4] propose several focus group designs, which include single-category, multiple-category, double-layer and broad-involvement designs. In our study, we initially aimed for a double-layer design and thought of a quantitative way on how to sample participants with several different characteristics within one group. However, as attrition cannot be planned beforehand, we discover that single-category designs are the preferred design choice for online focus groups. We learned from our online focus groups and other studies (e.g., [5]), that it does make sense to use a pre-screening survey to determine the potential participant's fit, availability, demographics, and consent.

Additionally, we would like to follow others in recommending substantial over-recruitment [7], [9], [12]. While group composition can hardly be pre- determined, efforts should be taken to influence the number of participants. We regard two participants as minimum for an online focus group, which distinguishes them from individual interviews. While face-to-face focus groups recommend group sizes of up to 12 participants [2], online focus groups benefit from smaller group sizes [9]. In our online focus groups, the discussion with two to four participants was much more insightful than the one with seven participants. This was due to longer and more frequent talking opportunities of each individual participant, which engaged participants. In larger groups, each single participant has to wait considerably longer to speak which might evoke disinterest and fatigue.

Research setting: In prior literature, there is evidence that online focus groups result in data that is as rich as face-to-face focus groups [13]. To achieve data richness in online focus groups, particularities of videoconferencing technology have to be considered. While there are advantages and disadvantages of specific videoconferencing technology, contemporary technology inexpensively meet online focus group requirements, such as capacities for multiple participants, no signup requirements for participants, breakout rooms, video and audio transmission, chat, and recording functionalities [9].

In prior literature, frequently reported technical challenges include delayed connectivity, speaker and microphone adjustments, inconsistent sound quality and technical interruptions [9]. We experienced only one instance in which these problems considerably disrupted the group discussion. One of two participants in an online focus group had a bad internet connection and dropped out for about 30 minutes. During this time, we interviewed the remaining participant alone. As soon as the other participant returned, we were already finished with the first individual interview. Thus, we continued the discussion with the returning participant where he broke off and also interviewed him separately. Therefore, individual online interviews, rather than online focus groups, were conducted.

These kinds of technical disruptions cannot be prevented, and the moderator has to manage the issue ad hoc. One potential mitigation is a group size larger than two. It is highly unlikely that any two participants experience technical issues at the exact same time. If there are always at least two other participants present, the discussion can continue, and the moderator can reintegrate the returning participants when they are back.

Apart from such intrusive disruptions, handling the technology itself can prove difficult for participants [12]. One option to support participants is to provide them with the opportunity to test the software beforehand [7]. In our online focus groups, we found it to be sufficient to tell the participants prior to the online 
focus group which videoconferencing tool will be used and to provide them with a link to the guide of the software provider. Additionally, before the online focus group started, research assistants answered all technical questions of the participants. None of our participants expressed or showed any insecurity with regards to the technology in the online focus group discussion, although first-time users were present. This might be different if other participants, who are not used to interact with digital technology on a daily basis, join online focus groups.

Another helpful aspect might be to ask a research assistant to be present throughout the whole online focus group, such that she/ he can discuss individual technical difficulties with participants outside the main discussion room. While we asked a research assistant to be present during the pilot online focus group, we never needed his service and decided to conduct the following online focus groups without his assistance. However, we might have been lucky. Others also report to have an assistant on standby [7], who can spontaneously jump in in case a participant experiences technical difficulties.

Apart from the participants, researchers might also be inexperienced with videoconferencing technology. Thus, prior studies recommend spending considerable time to familiarize themselves with the technology and conduct practice rounds [7], [9], [12]. As our researcher team was used to videoconferencing technology due to experience with it in teaching, we did not conduct practice rounds to test the tools.

As participants can decide on where to join the online focus group, the choice of their research location is out of the researcher's control. We were concerned that participants might join in from inappropriate locations, such as while driving or in noisy environments. Therefore, we asked them in prior communication via email/ messages to make sure that their environment is safe and without disruptions to the online focus group. During the online focus groups, the research assistants took note of the participants' environments as well. All of the participants seemed to be at a home and, although pets and children were present, there was no notable disruption caused by the environment of the participants. Thus, we regard a note on the appropriateness of the environment as sufficient to address the challenge of inappropriate research settings.

Preparation of the online focus group: Online focus groups provide for more anonymity of participants, which raises concerns for researchers regarding the identity of participants [12]. Additionally, due to the lack of possibilities for private conversations, the preparation of online focus groups differs from that of face-to-face focus groups. The latter implies that sideconversations, individual questions and requests, and socializing among the participants, as well as between participants and moderators cannot easily take place.

While doubts in the identity of participants are more likely in text-based online focus groups than in videobased online focus groups, it is still necessary to make sure that the selected participants participate. In our study, we maintained contact via the same email address/ instant messaging account throughout the screening survey, the notification of acceptance, the reminders, and the payment. Additionally, we asked participants in the individual welcome part to tell us their email address/ instant messaging account and compared it to the previous one. None of the participants failed this check and we never had any doubts in the discussion that participants were not as knowledgeable as they claimed to be beforehand.

In order to overcome the challenge that multiple individual conversations cannot take place concurrently during the online focus groups, we took two measures. First, between the participants' declaration of interest, and the start of the online focus group, we regularly engaged with them, which is in line with best practice [9]. In doing so, rapport-building has to be carefully weighted with perceptions of privacy intrusion [12]. In all of our emails/ messages we presented the opportunity for participants to raise questions, such that we were able to address any open questions or concerns before the online focus group took place. If they did not have any requests, participants received three preparation emails/ messages from us: automatic screening survey response, invitation, and reminder. None of the participants seem to have perceived this as intrusive.

Second, each participant was welcomed individually. Thus, individualized and private issues, such as technical checks, consent to recording and the payment process for the compensation could be relocated to individual sessions. This allowed the participants to address pressing organizational issues and individual questions upfront and separate these organizational issues from the main group discussion. Thus, neither the other participants, nor the moderator were distracted from topics outside the main discussion.

An additional idea that we had, but did not implement, is to offer networking opportunities. Participants could stay in the videoconferencing room after the online focus group to engage in individual discussions and exchange private contact information.

In order for the participants to prepare for the group discussion, one participant expressed the wish to receive the main questions prior to the online focus group. While this would reduce spontaneity, it might yield more information during the online focus group.

Facilitation of the online focus group: The role of the moderator is critical for an insightful group discussion [6]. In online focus groups, the technology 
has an influence on how the moderator can facilitate the discussion. While focus groups generally benefit from having only one person speak at a time [1], this might inhibit the flow of the communication and reduce spontaneity of responses [12]. In Matthews et al.'s study [7], four online focus group participants indicated that they felt that the discussion did not flow as easily as in face-to-face focus groups. Reduced intimacy and emotional detachment of the moderator when discussing highly emotional topics might also be additional challenges for online focus group moderators [7], [12]. However, no sensitive atmosphere arose in our online focus groups.

In prior literature on online focus groups, the moderator's role is generally perceived to be similar to their role in face-to-face focus groups [7], [9]. However, in order to maintain a steady flow of communication, it is also advised that the moderator takes on a more active role [12]. We agree with the latter view. While participants built on each other's comments and addressed each other directly, generally, the expectation seemed to be to be called upon by the researcher. This might be due to politeness and a wish to not disrupt the previous speaker. Thus, we recommend that the moderator takes on a more active role in the moderation of the online focus group.

Additionally, Stewart and Shamdasani [12] highlight the potential of technology through timetracking monitors and hand raise functionalities in assisting the moderator. These can be implemented to ensure that all participants receive similar attention and contribute to the online focus group. Attention has to be taken though, such that the online focus group does not turn into a more structured group interview [5]. One of our participants suggested that the moderator's screen could be shared, such that questions and notes would be visible to all participants.

Follow-up: In face-to-face focus groups, participants are debriefed, paid and bade goodbye at the end of the focus group itself [4]. However, similar to the preparation of online focus groups, the lack of possibilities for private conversations prevents these kinds of conversations in online focus groups. Thus, we propose the addition of a follow-up step in online focus groups. It involves arranging for individual contact with each participant after the online focus group.

While individual follow-up meetings with international participants originated from administrative reasons, they turned out to be valuable for two reasons. First, researchers receive an additional point of contact. Rather than making post-hoc sense of ambiguous statements participants expressed during the online focus group, researchers can ask clarification and follow-up questions. Second, participants receive the opportunity to use the time after the online focus group to reflect on their contributions and experiences. They can also pose additional questions, e.g., regarding payment, which officially closes the online focus group process for them.

\section{Limitations and future research}

The limitations of focus groups and online focus group are presented below. Additionally, we outline the limitations of our own analysis of online focus groups and propose future research to advance the method.

Focus groups possess some inherent limitations, just like any other research method [1]. Some of these limitations cannot be overcome with online focus groups. They cannot be used to generate statistically significant explanations or predictions [2], [4], [6]. Samples are usually small, not representative and selfselected and convenience samples [1], [2]. However, they are useful for a broad range of purposes which we outlined above.

Although we presented possible solutions to challenges of online focus groups, there remain some limitations. While the internet is broadly available, people without internet access and technology access to video-conferencing software and hardware cannot participate in online focus groups [12]. Language barriers might exist, which prevents certain groups of people from participating. Although online focus groups allow engaging a wider group of participants, sampling may still be restricted by technical issues, time differences, and attrition cannot be ruled out.

In our analysis, we focused on video-based online focus groups, which constitute an emerging way of conducting online focus groups. However, we outlined above their high potential for valuable contributions in IS research. Our analysis of online focus groups involves all steps which differ from face-to-face focus groups. However, we did not discuss data analysis and reporting, which are critical for any research method [20]. For excellent discussions of focus group analysis, we refer the reader to other sources (e.g., [2], [5], [21][23]). With regards to reporting, we note that the topic has been neglected in IS focus group research (see our literature review where only $20 \%$ of papers reported on their focus groups in detail). While reporting principles for focus groups can be found in Krueger and Casey [4], we believe that reporting standards for online focus groups will evolve over time with their increased application in IS research.

Furthermore, while we have substantial experience with the focus group method itself, and online focus groups in particular, collectively, our field has more experience. We hope that our paper attracts the attention of IS researchers who conduct online focus groups and are interested in a deeper discussion about the method. 
This would help us in triangulating our own experiences with those of others in order to increase the robustness and value of our recommendations.

\section{Conclusion}

Online focus groups bear the potential to generate rich insights into many IS phenomena by overcoming some of the challenges of face-to-face focus groups. We outline the conditions for the nature of the phenomena and the nature of the participants that determine the appropriateness of online focus groups. The report of our own experiences showcases the challenges of online focus groups. We discuss potential solutions to those challenges in the identification and recruitment of participants, the research setting, the preparation, facilitation, and follow-up of the online focus group. Thereby, IS researchers are provided with practical advice on how to overcome the challenges of designing and conducting online focus groups. The prospect of an increasing number of online focus groups in the future will determine the usefulness of these best practices and open up a discourse on advancements of the online focus group method.

\section{References}

[1] E. F. Fern, Advanced Focus Group Research, Sage, California, 2001.

[2] S. Wilkinson, "Focus Group Methodology: A Review," International Journal of Social Research Methodology, 1998, pp. 181-203.

[3] F. Bélanger, "Theorizing in Information Systems Research Using Focus Groups," Australasian Journal of Information Systems, 2012, pp. 109-135.

[4] R. A. Krueger and M. A. Casey, Focus Groups: A Practical Guide for Applied Research, 5th edition., Sage, California, 2014.

[5] A. Parker and J. Tritter, "Focus Group Method and Methodology: Current Practice and Recent Debate," International Journal of Research \& Method in Education, 2006, pp. 23-37.

[6] T. O.Nyumba, K. Wilson, C. J. Derrick, and N. Mukherjee, "The Use of Focus Group Discussion Methodology: Insights from Two Decades of Application in Conservation," Methods in Ecology and Evolution, 2018, pp. 20-32.

[7] K. L. Matthews, M. Baird, and G. Duchesne, "Using Online Meeting Software to Facilitate Geographically Dispersed Focus Groups for Health Workforce Research," Qualitative Health Research, 2018, pp. 1621-1628.
[8] D. L. Clapper and A. P. Massey, "Electronic Focus Groups: A Framework for Exploration," Information \& Management, 1996, pp. 43-50.

[9] C. A. Tuttas, "Lessons Learned Using Web Conference Technology for Online Focus Group Interviews," Qualitative Health Research, 2015, pp. 122-133.

[10] J. Van Maanen, "Confessional Tales," in Tales of the Field: On Writing Ethnography, 2nd ed., Chicago: University of Chicago Press, 2011, pp. 73-100.

[11] Gregor, "The Nature of Theory in Information Systems,” MIS Quarterly, 2006, pp.611-642.

[12] D. W. Stewart and P. Shamdasani, "Online Focus Groups," Journal of Advertising, 2017, pp. 48-60.

[13] K. M. Abrams, Z. Wang, Y. J. Song, and S. GalindoGonzalez, "Data Richness Trade-Offs Between Face-toFace, Online Audiovisual, and Online Text-Only Focus Groups," Social Science Computer Review, 2015, pp. 80-96.

[14] B. Bergvall-Kåreborn and D. Howcroft, "Persistent Problems and Practices in Information Systems Development: A Study of Mobile Applications Development and Distribution," Information Systems Journal, 2014, pp. 425-444.

[15] A. Sidorova, N. Evangelopoulos, J. S. Valacich, and T. Ramakrishnan, "Uncovering the Intellectual Core of the Information Systems Discipline,” MIS Quarterly, 2008, pp. 467-482.

[16] A. Burton-Jones, B. S. Butler, S. Scott, and S. X. Xu, "Next-Generation Information Systems Theorizing: A Call to Action,” MIS Quarterly, 2021, pp. 301-314.

[17] D. Leidner and O. Tona, "The CARE Theory of Dignity Amid Personal Data Digitalization," MIS Quarterly, 2021, pp. 343-370.

[18] A. Rai, P. Constantinides, and S. Sarker, "Editor's Comments: Next-Generation Digital Platforms: Toward Human-AI Hybrids,” MIS Quarterly, 2019, pp. iii-ix.

[19] U. Rani and M. Furrer, "Digital Labour Platforms and New Forms of Flexible Work in Developing Countries: Algorithmic Management of Work and Workers," Competition \& Change, 2020, pp. 1-24.

[20] K. Conboy, G. Fitzgerald, and L. Mathiassen, "Qualitative Methods Research in Information Systems: Motivations, Themes, and Contributions," European Journal of Information Systems, 2012, pp. 113-118.

[21] A. J. Onwuegbuzie, W. B. Dickinson, N. L. Leech, and A. G. Zoran, "A Qualitative Framework for Collecting and Analyzing Data in Focus Group Research," International Journal of Qualitative Methods, 2009, pp. $1-21$.

[22] J. Sim, "Collecting and Analysing Qualitative Data: Issues Raised by the Focus Group," Journal of Advanced Nursing, 1998, pp. 345-352.

[23] P. S. Kidd and M. B. Parshall, "Getting the Focus and the Group: Enhancing Analytical Rigor in Focus Group Research," Qualitative Health Research, 2000, pp. 293 308. 